\title{
A Matrix Interpretation of the Extended Euclidean Algorithm
}

\section{Report}

Author(s):

Gutknecht, Martin H.

Publication date:

2000-05

Permanent link:

https://doi.org/10.3929/ethz-a-004288272

Rights / license:

In Copyright - Non-Commercial Use Permitted

Originally published in:

SAM Research Report 2000-05 


\title{
A Matrix Interpretation of the Extended Euclidean Algorithm*
}

\author{
M.H. Gutknecht
}

Research Report No. 2000-05

May 2000

Seminar für Angewandte Mathematik Eidgenössische Technische Hochschule CH-8092 Zürich

Switzerland

\footnotetext{
* To appear in "Structured Matrices in Operator Theory, Numerical Analysis, Control, Signal and Image Processing" (V. Olshevsky, ed.), Contemporary Mathematics Series, American Math. Society
} 


\title{
A Matrix Interpretation of the Extended Euclidean Algorithm*
}

\author{
M.H. Gutknecht \\ Seminar für Angewandte Mathematik \\ Eidgenössische Technische Hochschule \\ CH-8092 Zürich \\ Switzerland
}

Research Report No. 2000-05

May 2000

\begin{abstract}
The extended Euclidean algorithm for polynomials and formal power series that is used for the recursive computation of Padé approximants can be viewed in various ways as a sequence of successive matrix multiplications that are applied to a Sylvester matrix with the original data. Here we present this result in a general version that includes the treatment of the Cabay-Meleshko look-ahead algorithm, which generalizes the extended Euclidean algorithm and yields a weakly stable (forward stable) method for computing Padé fractions if it is combined with an appropriate rule for choosing the look-ahead step length. Moreover, we choose for the matrix interpretation a particularly appealing form where also the product of all the matrices that are applied has a meaning: this product yields at the end four Toeplitz blocks with the coefficients of the polynomials (which belong to Padé forms) that are generated by the extended Euclidean algorithm in addition to those resulting from the ordinary Euclidean algorithm.
\end{abstract}

Subject Classification: Primary 41A21; Secondary 30E05

* To appear in "Structured Matrices in Operator Theory, Numerical Analysis, Control, Signal and Image Processing" (V. Olshevsky, ed.), Contemporary Mathematics Series, American Math. Society 


\section{Introduction and notation}

The Euclidean algorithm for polynomials or power series is closely related to Padé approximation. It is, in fact, up to minor modifications, one of the standard algorithms to compute Padé approximants along a diagonal of the Padé table and to generate the corresponding continued fraction (called a P-fraction), although the name Euclid comes only up in part of the Padé literature. An excellent, detailed account of these connections is given by Bultheel and Van Barel [BV97]; some of the main aspects are also treated by Gragg and Gutknecht [GG94] and others.

Since the Euclidean algorithm (in the form discussed here) concerns linear combinations of polynomials and power series, it is no surprise that one can interpret it in terms of matrices. Recall that the ring of formal power series is isomorphic to the ring of infinite lower triangular Toeplitz matrices. Such matrix interpretations of the Euclidean algorithm for polynomials are not very common, but some sketches can be found in the literature, at least for the (generic) case where in each step the degrees of the polynomials reduce only by 1 ; see, for example, Householder and Stewart [HS69] - we do not attempt to give a complete set of references here. A single step of the algorithm is described as a matrix multiplication, and the whole algorithm takes the form of a matrix product consisting of a Sylvester matrix with the original data multiplied by a sequence of matrices that contain the quotients from the Euclidean algorithm. However, there is some freedom in arranging the data and in defining the factors of the product. We have succeeded to do this in a way so that the product of the matrix factors becomes a $4 \times 4$ block matrix with Toeplitz blocks that contain the coefficients of the Padé forms that are implicitly constructed in the process. In the terminology of Padé approximation, the Euclidean algorithm produces residuals of Padé approximants, while the Padé approximants itself, or rather the Padé forms (which are pairs of polynomials that are the numerators and denominators of the Padé approximants, respectively) are constructed additionally in the so-called extended Euclidean algorithm [BV97]. The matrix identity that we derive links the Sylvester matrix containing the given data not only to the recurrence coefficients (quotients) of the Euclidean algorithm but also with the six different sets of polynomials that are generated by the extended version. Moreover, the well-known symmetric LDU and inverse symmetric LDU decompositions of the Hankel moment matrix associated with the Padé problem can be read off the matrix identity.

Our motivation for this work came from section 9 of [GH95a], where, building up on earlier work by Delosme and Ipsen [DI89], Gutknecht and Hochbruck derived an analogous matrix representation of the look-ahead Levinson and Schur(-Bareiss) algorithms introduced in that paper. There too the resulting matrix identities can be interpreted in many ways. In particular, they are linked to the LDU and inverse LDU decomposition of the Toeplitz matrix of the given trigonometric moments.

Before we can start with the derivation and description of our matrix form of the Euclidean algorithm we present in sections 2-6 the prerequisites. This partly well-known material is extracted from [GG94] and suitably adapted to the situation and needs of this paper.

Notation. We let $\mathcal{L}$ be the set of formal Laurent series

$$
h_{0}(\zeta)=\sum_{k=-\infty}^{\infty} \mu_{k} \zeta^{k}
$$


with complex coefficients. The following subsets of $\mathcal{L}$ will play a role:

$$
\begin{aligned}
\mathcal{L}_{l: m} & : \equiv\left\{h_{0} \in \mathcal{L} ; \mu_{k}=0 \text { if } k<l \text { or } k>m\right\}, \\
\mathcal{L}_{m}^{*} & : \equiv \mathcal{L}_{-\infty: m}=\left\{h_{0} \in \mathcal{L} ; \mu_{k}=0 \text { if } k>m\right\}, \\
\mathcal{L}_{0}^{*} & : \equiv \mathcal{L}_{-\infty: 0}=\text { formal power series in } \zeta^{-1}, \\
\mathcal{P}_{m} & : \equiv \mathcal{L}_{0: m}=\text { polynomials in } \zeta \text { of degree } m, \\
\mathcal{P} & : \equiv \mathcal{U} \mathcal{P}_{m}=\text { polynomials in } \zeta .
\end{aligned}
$$

For $h_{0} \in \mathcal{L}_{m}^{*}$ or $h_{0} \in \mathcal{P}$ we define the exact degree $\partial h_{0}$ by

$$
\partial h_{0}: \equiv\left\{n \in \mathbb{Z} ; \mu_{n} \neq 0, \mu_{k}=0 \text { for } k>n\right\} .
$$

We also use the notation $h_{0}(\zeta)=\mathcal{O}_{-}\left(\zeta^{m}\right)$ to indicate that $h_{0} \in \mathcal{L}_{m}^{*}$, while $h_{0}(\zeta) \equiv$ $\mathcal{O}_{-}\left(\zeta^{m}\right)$ means that $h_{0}(\zeta) \in \mathcal{L}_{m}^{*}$, but $h_{0}(\zeta) \notin \mathcal{L}_{m-1}^{*}$; in other words, it means the same as $\partial h_{0}=m$. Moreover, if $h_{0} \in \mathcal{L}_{0}^{*}$ we define $h_{0}(\infty): \equiv \mu_{0}$.

Finally, $\mathcal{R}_{m, n}$ denotes the set of rational functions of type $(m, n)$. Such a function $r$ that can be represented as the quotient of two not necessarily relatively prime polynomials, $r=p / q$, where the numerator $p$ has degree at most $m$, and the denominator $q$ has degree at most $n$ and is not the zero polynomial.

While $: \equiv$ is used to denote definitions, $:=$ indicates assignments that may be used in an algorithm. (Sometimes either one could be applied.)

\section{Padé approximants of a pair of formal power series}

Given a pair of formal power series in $\zeta^{-1}$,

$$
\begin{array}{lll}
f_{0} \in \mathcal{L}_{-1}^{*}, & f_{0} \neq 0, & f_{0}(\zeta) \equiv: \sum_{j=1}^{\infty} \phi_{j, 0} \zeta^{-k}, \\
g_{0} \in \mathcal{L}_{0}^{*}, & g_{0}(\infty)=1, & g_{0}(\zeta) \equiv: \sum_{j=0}^{\infty} \gamma_{j, 0} \zeta^{-k},
\end{array}
$$

we consider, for fixed $n>1$, pairs of pairs of polynomials,

$$
\left(\grave{p}_{n}, \grave{q}_{n}\right) \in \mathcal{P}_{n-2} \times \mathcal{P}_{n-1}, \quad\left(p_{n}, q_{n}\right) \in \mathcal{P}_{n-1} \times \mathcal{P}_{n} \quad \text { with } q_{n} \text { monic, }
$$

satisfying the following conditions: there are two new formal power series in $\zeta^{-1}$ of the form

$$
f_{n} \in \mathcal{L}_{-n-1}^{*}, \quad g_{n} \in \mathcal{L}_{-n}^{*} \quad \text { with } g_{n}(\zeta)=\zeta^{-n}+\mathcal{O}_{-}\left(\zeta^{-n-1}\right)
$$

such that

$$
\begin{aligned}
& \underbrace{g_{0}(\zeta) \grave{p}_{n}(\zeta)}_{\in \mathcal{L}_{n-2}^{*}}+\underbrace{f_{0}(\zeta) \grave{q}_{n}(\zeta)}_{\in \mathcal{L}_{n-2}^{*}}=\underbrace{g_{n}(\zeta)}_{\in \mathcal{L}_{-n}^{*}}, \\
& \underbrace{g_{0}(\zeta) p_{n}(\zeta)}_{\in \mathcal{L}_{n-1}^{*}}+\underbrace{f_{0}(\zeta) q_{n}(\zeta)}_{\in \mathcal{L}_{n-1}^{*}}=\underbrace{f_{n}(\zeta)}_{\in \mathcal{L}_{-n-1}^{*}} .
\end{aligned}
$$

This means that $\left(p_{n}, q_{n}\right)$ and $\left(\grave{p}_{n}, \grave{q}_{n}\right)$ are Padé forms of the pair $\left(g_{0}, f_{0}\right)$ at $\zeta=\infty$. The Padé form $\left(p_{n}, q_{n}\right)$ consists of the numerator $p_{n}$ and the denominator $q_{n}$ of the $(n, n)$ Padé approximant $r_{n, n}: \equiv p_{n} / q_{n}$, and, likewise, $\left(\grave{p}_{n}, \grave{q}_{n}\right)$ contains the (differently normalized, not necessarily mutually prime) numerator and denominator of a $(n-1, n-1)$ Padé approximant, if we consider the fractions as functions of $\zeta^{-1}$. Therefore, these Padé approximants lie on the main diagonal of the Padé table of $\left(g_{0}, f_{0}\right)$. We call the formal power series $g_{n}$ and $f_{n}$ in $\zeta^{-1}$ residuals of $\left(p_{n}, q_{n}\right)$ and $\left(\grave{p}_{n}, \grave{q}_{n}\right)$, respectively. (In the literature, often the shifted series $\zeta^{n} g_{n}(\zeta)$ and 
$\zeta^{n} f_{n}(\zeta)$ are referred to as the residuals; but here the formulas will be simpler when we let the leading $n$ or $n+1$ coefficients be zero.)

Note that (2.4a) implies that the $2 n-2$ coefficients of the terms $\zeta^{-n+1}, \ldots, \zeta^{n-2}$ on the left-hand side must be set to 0 , and by (2.3) the one of $\zeta^{-n}$ is 1 . These $2 n-1$ conditions are matched by the same number of degrees of freedom in the coefficients of $\grave{p}_{n}$ and $\grave{q}_{n}$. Likewise, in $(2.4 \mathrm{~b})$ the $2 n$ coefficients of the terms $\zeta^{-n}, \ldots, \zeta^{n-1}$ are zero, and the polynomials $p_{n}$ and $q_{n}$ have exactly $2 n$ free coefficients since $q_{n}$ must be monic.

Normally, Padé approximation is defined for a single formal power series. We can return to this situation by noting that due to $g_{0}(\infty)=1$ the quotient $h_{0}(\zeta): \equiv$ $-f_{0}(\zeta) / g_{0}(\zeta) \in \mathcal{L}_{-1}^{*}$ is well defined. If we then redefine in the above formulas

$$
g_{0}(\zeta): \equiv 1, \quad f_{0}(\zeta): \equiv-h_{0}(\zeta),
$$

it is easy to verify that $(2.4 \mathrm{a})-(2.4 \mathrm{~b})$ match with the usual Padé conditions. The series

$$
h_{0}(\zeta) \equiv: \sum_{k=1}^{\infty} \frac{\mu_{k}}{\zeta^{k}} \in \mathcal{L}_{-1}^{*},
$$

is the generating function of the Markov parameters or moments $\left\{\mu_{k}\right\}_{k=1}^{\infty}$ and can be viewed as the symbol of an infinite Hankel matrix $\mathrm{M}$ defined by $m_{k, l}: \equiv \mu_{k+l-1}$.

It is well known and easy to verify, see for example [GG94], that unique solutions $\left(\grave{p}_{n}, \grave{q}_{n}\right)$ and $\left(p_{n}, q_{n}\right)$ to the conditions $(2.2)-(2.4 \mathrm{~b})$ exist if and only if the $n$th principal leading submatrix

$$
\mathrm{M}_{n}:=\left[\begin{array}{cccc}
\mu_{1} & \mu_{2} & \cdots & \mu_{n} \\
\mu_{2} & & . \cdot & \vdots \\
\vdots & . \cdot & & \vdots \\
\mu_{n} & \cdots & \cdots & \mu_{2 n-1}
\end{array}\right]
$$

of $\mathrm{M}$ is nonsingular. In this case, we call $q_{n}$ a regular formal orthogonal polynomial or regular FOP, the pair of denominators $\left(\grave{q}_{n}, q_{n}\right)$ a normalized $n$th regular pair and $n$ a regular index. Heinig and Rost [HR84] refer to the pair as fundamental solutions, since it allows them to give an explicit formula for $\mathrm{M}^{-1}$. Note that in the situation (2.5) it is trivial to determine the numerators $\grave{p}_{n}$ and $p_{n}$ once the denominators $\grave{q}_{n}$ and $q_{n}$ are known.

\section{The recursive computation of Padé approximants by a generalized extended Euclidean algorithm}

By using a $2 \times 2$ matrix with polynomial entries and row vectors with formal power series as entries we can write (2.4a) $-(2.4 \mathrm{~b})$ as

$$
\left[\begin{array}{ll}
g_{0} & f_{0}
\end{array}\right]\left[\begin{array}{cc}
\grave{p}_{n} & p_{n} \\
\grave{q}_{n} & q_{n}
\end{array}\right]=\left[\begin{array}{ll}
g_{n} & f_{n}
\end{array}\right]
$$

Note that if we extracted from the row vector on the right-hand side the common factor $\zeta^{-n}$, the remainder would be of the same form as the row vector [ $\left.\begin{array}{ll}g_{0} & f_{0}\end{array}\right]$. This indicates, but does not prove yet, that we can set up a recursive procedure. One still has to show that the corresponding $2 \times 2$ matrices fit together appropriately.

Generalizing earlier work of Gragg, Gustavson, Warner, and Yun [GGWY82] that was restricted to the generic case (where all leading principal submatrices of 
$\mathrm{M}$ are nonsingular) Cabay and Meleshko [CM93] established stable recurrences of this sort for computing well-conditioned Padé approximants along a diagonal of the Padé table. Here, well-conditioned means that the submatrix $\mathrm{M}_{n}$, which could alternatively be used to determine these Padé approximants, is in a certain sense (left vague here) well conditioned. These Cabay-Meleshko recurrences are a special case of the following theorem, which could be used to compute any ordered sequence of regular pairs along the main diagonal of the table. The generalization to other diagonals is trivial. In the current notation this theorem is given and proved in [GG94].

THEOREM 3.1. Let $\left[\begin{array}{ll}\grave{q}_{n} & q_{n}\end{array}\right]$ be a normalized nth regular pair for $\left[\begin{array}{ll}g_{0} & f_{0}\end{array}\right]$, so that (3.1) holds with (2.2)-(2.3). Likewise, let [ $\grave{a} a$ ] be a normalized kth regular pair for $\zeta^{n}\left[\begin{array}{ll}g_{n} & f_{n}\end{array}\right]$, so that

$$
(\grave{b}, \grave{a}) \in \mathcal{P}_{k-2} \times \mathcal{P}_{k-1}, \quad(b, a) \in \mathcal{P}_{k-1} \times \mathcal{P}_{k} \quad \text { with a monic, }
$$

and the residuals

$$
\left[\begin{array}{ll}
g_{n+k} & f_{n+k}
\end{array}\right]:=\left[\begin{array}{ll}
g_{n} & f_{n}
\end{array}\right]\left[\begin{array}{cc}
\grave{b} & b \\
\grave{a} & a
\end{array}\right]
$$

satisfy

$$
f_{n+k} \in \mathcal{L}_{-n-k-1}^{*}, \quad g_{n+k} \in \mathcal{L}_{-n-k}^{*}
$$

with

$$
g_{n+k}(\zeta)=\zeta^{-n-k}+\mathcal{O}_{-}\left(\zeta^{-n-k-1}\right) .
$$

Then

$$
\left[\begin{array}{cc}
\grave{p}_{n+k} & p_{n+k} \\
\grave{q}_{n+k} & q_{n+k}
\end{array}\right]:=\left[\begin{array}{cc}
\grave{p}_{n} & p_{n} \\
\grave{q}_{n} & q_{n}
\end{array}\right]\left[\begin{array}{cc}
\grave{b} & b \\
\grave{a} & a
\end{array}\right]
$$

yields a normalized $(n+k)$ th regular pair $\left[\begin{array}{ll}\grave{q}_{n+k} & q_{n+k}\end{array}\right]$ for $\left[\begin{array}{ll}g_{0} & f_{0}\end{array}\right]$ as well as the corresponding numerators [ $\grave{p}_{n+k} \quad p_{n+k}$ ].

The corresponding pair of residuals is $\left[\begin{array}{ll}g_{n+k} & f_{n+k}\end{array}\right]$. Hence, (3.3) is the recurrence for the residuals.

To obtain stable recurrences one needs a rule for identifying well-conditioned normalized regular pairs; see Cabay and Meleshko [CM93] and, for further results and comments on this and related matter, Beckermann [Bec96] and Gragg and Gutknecht [GG94]. The basis of this rule is Heinig's inversion formula for $\mathbf{M}_{n}$, an improved variation of the well-known Gohberg-Semencul formula; see Heinig and Rost [HR84] and Gutknecht and Hochbruck [GH95b].

The condition (3.3) for the "recurrence coefficients", that is, the Padé forms $(\grave{b}, \grave{a})$ and $(b, a)$, translates easily into a pair of linear systems for the coefficients of these Padé forms. Let

$$
g_{n}(\zeta) \equiv: \sum_{j=0}^{\infty} \gamma_{j, n} \zeta^{-n-j}, \quad f_{n}(\zeta) \equiv: \sum_{j=1}^{\infty} \phi_{j, n} \zeta^{-n-j},
$$


with $\gamma_{0, n}=1$, and consider the $2 k \times 2 k$ Sylvester matrix

$$
\mathrm{S}_{k}\left(g_{n}, f_{n}\right): \equiv\left[\begin{array}{cccc|cccc}
0 & \cdots & 0 & 1 & 0 & \cdots & \cdots & 0 \\
\vdots & . & 1 & \gamma_{1, n} & \vdots & & 0 & \phi_{1, n} \\
0 & . & . \cdot & \vdots & \vdots & . \cdot & . \cdot & \vdots \\
1 & . & & \gamma_{k-1, n} & 0 & . \cdot & & \phi_{k-1, n} \\
\gamma_{1, n} & & . & \gamma_{k, n} & \phi_{1, n} & & . & \phi_{k, n} \\
\vdots & . & . & \vdots & \vdots & . \cdot & . \cdot & \vdots \\
\gamma_{k-1, n} & . & & \vdots & \phi_{k-1, n} & . & & \vdots \\
\gamma_{k, n} & \cdots & \cdots & \gamma_{2 k-1, n} & \phi_{k, n} & \cdots & \cdots & \phi_{2 k-1, n}
\end{array}\right] .
$$

Moreover, set

$$
\mathrm{e}_{2 k, 2 k}: \equiv\left[\begin{array}{cccc}
0 & \cdots & 0 & 1
\end{array}\right]^{\top} \in \mathbb{C}^{2 k}, \quad \mathrm{f}_{2 k, n}: \equiv-\left[\phi_{1, n} \cdots \phi_{2 k, n}\right]^{\top},
$$

and let

$$
\grave{\mathrm{b}}, \quad \grave{\mathrm{a}}, \quad \mathrm{b} \in \mathbb{C}^{k}, \quad \mathrm{a}=\left[\begin{array}{c}
\dot{\mathrm{a}} \\
1
\end{array}\right] \in \mathbb{C}^{k+1}
$$

be the coefficient vectors of the polynomials $\grave{b}, \grave{a}, b$, and $a$, the first one being augmented by a zero component. Then (3.3) becomes, when the last component 1 of a is moved to the right-hand side,

$$
\mathrm{S}_{k}\left(g_{n}, f_{n}\right)\left[\begin{array}{ll}
\grave{b} & \mathrm{~b} \\
\grave{a} & \grave{a}
\end{array}\right]=\left[\begin{array}{ll}
\mathrm{e}_{2 k, 2 k} & \mathrm{f}_{2 k, n}
\end{array}\right] .
$$

When $k=1$, which is the generic case, (3.11) reduces to

$$
\left[\begin{array}{cc}
1 & 0 \\
\gamma_{1, n} & \phi_{1, n}
\end{array}\right]\left[\begin{array}{cc}
\grave{\beta}_{0} & \beta_{0} \\
\grave{\alpha}_{0} & \alpha_{0}
\end{array}\right]=\left[\begin{array}{cc}
0 & -\phi_{1, n} \\
1 & -\phi_{2, n}
\end{array}\right]
$$

from which it follows that

$$
\grave{\beta}_{0}=0, \quad \grave{\alpha}_{0}=\frac{1}{\phi_{1, n}}, \quad \beta_{0}=-\phi_{1, n}, \quad \alpha_{0}=\gamma_{1, n}-\frac{\phi_{2, n}}{\phi_{1, n}} .
$$

\section{J-fractions, P-fractions, and the extended Euclidean algorithm for power series}

The recurrences of Theorem 3.1 are very general, and it is not so obvious what they have in common with the Euclidean algorithm. To explain this, we consider here two special cases, namely the one where $k=1$ for all $n$, and thus all $n$ are regular indices, and the one where $k>1$ is allowed, but $n$ and $n+k$ are two successive regular indices.

Therefore, for a moment assume that for all $n, q_{n}$ is a regular FOP. Then the recursion (3.6) can be applied for all $n$ with $k=1$, where the coefficients are given by (3.13). Recall that this holds, if and only if $\phi_{1, n} \neq 0$ for all $n$. Then

$$
\left(\grave{p}_{n+1}, \grave{q}_{n+1}\right)=\left(p_{n}, q_{n}\right) / \phi_{1, n},
$$


and hence

$$
\left[\begin{array}{cc}
p_{n} & p_{n+1} \\
q_{n} & q_{n+1}
\end{array}\right]:=\left[\begin{array}{cc}
p_{n-1} & p_{n} \\
q_{n-1} & q_{n}
\end{array}\right]\left[\begin{array}{cc}
0 & -\beta_{n+1} \\
1 & \zeta-\alpha_{n+1}
\end{array}\right]
$$

where

$$
\beta_{n+1}:=\frac{\phi_{1, n}}{\phi_{1, n-1}}, \quad \alpha_{n+1}:=\frac{\phi_{2, n}}{\phi_{1, n}}-\gamma_{1, n},
$$

with $\phi_{1,-1}:=-1, \phi_{k, 0}:=\phi_{k}, \gamma_{k, 0}:=\gamma_{k}$.

Eliminating the trivial part of (4.2) we find the well-known generic diagonal recurrences for Padé forms and FOPs,

$$
\begin{aligned}
& p_{n}(\zeta):=\left(\zeta-\alpha_{n}\right) p_{n-1}(\zeta)-\beta_{n} p_{n-2}(\zeta), \\
& q_{n}(\zeta):=\left(\zeta-\alpha_{n}\right) q_{n-1}(\zeta)-\beta_{n} q_{n-2}(\zeta),
\end{aligned}
$$

with initial values

$$
\left(p_{-1}(\zeta), q_{-1}(\zeta)\right): \equiv(1,0), \quad\left(p_{0}(\zeta), q_{0}(\zeta)\right): \equiv(0,1) .
$$

They reveal that $p_{n}$ and $q_{n}$ are the $n$th numerator and denominator of the Jacobi fraction (J-fraction) of $h_{0}=-f_{0} / g_{0}$,

$$
h_{0}(\zeta)=-\frac{f_{0}(\zeta)}{g_{0}(\zeta)}=\frac{\beta_{1}}{\zeta-\alpha_{1}}-\frac{\beta_{2}}{\zeta-\alpha_{2}}-\cdots,
$$

that is,

$$
\frac{p_{n}(\zeta)}{q_{n}(\zeta)}=\frac{\beta_{1}}{\zeta-\alpha_{1}}-\cdots-\frac{\beta_{n}}{\zeta-\alpha_{n}} .
$$

This J-fraction does not exist if $\phi_{1, n}=0$ for some $n$. It may have very large $\left|\alpha_{n+1}\right|$ and $\left|\beta_{n+2}\right|$ if $\left|\phi_{1, n}\right|$ is small.

In exact arithmetic the case $\phi_{1, n}=0$ can be treated as follows: if

$$
\phi_{0, n}=\cdots=\phi_{k-1, n}=0, \quad \phi_{k, n} \neq 0,
$$

then $\mathrm{S}_{1}, \ldots, \mathrm{S}_{k-1}$ are singular, but $\mathrm{S}_{k}$ is nonsingular. The left system in (3.11) has then the solution

$$
\grave{b}(\zeta) \equiv 0, \quad \grave{a}(\zeta) \equiv \frac{1}{\phi_{k, n}} .
$$

Consequently,

$$
\left(\grave{p}_{n+k}, \grave{q}_{n+k}\right)=\frac{1}{\phi_{k, n}}\left(p_{n}, q_{n}\right), \quad g_{n+k}=\frac{\zeta^{k} f_{n}}{\phi_{k, n}} .
$$

In this way, we can proceed from any regular Padé form $\left(p_{n}, q_{n}\right)$ and its upperleft neighbor $\left(\grave{p}_{n}, \grave{q}_{n}\right)$ to the next one, $\left(p_{n+k}, q_{n+k}\right)$, and its upper-left neighbor $\left(\grave{p}_{n+k}, \grave{q}_{n+k}\right)$, where $k$ is determined by (4.8).

Let $\left\{n_{j}\right\}_{j=0}^{J}(J \leq \infty)$ be the sequence of all regular indices, starting with $n_{0}=0$, and let

$$
k_{j}: \equiv n_{j+1}-n_{j} \quad(j \geq 1) .
$$

Additionally, we set $n_{-1}:=-1, k_{0}:=1$. Then, $\phi_{k_{j}, n_{j}} \neq 0(\forall j)$. In analogy to (4.2), recurrence (3.6) becomes

$$
\left[\begin{array}{ll}
p_{n_{j}} & p_{n_{j+1}} \\
q_{n_{j}} & q_{n_{j+1}}
\end{array}\right]:=\left[\begin{array}{ll}
p_{n_{j-1}} & p_{n_{j}} \\
q_{n_{j-1}} & q_{n_{j}}
\end{array}\right]\left[\begin{array}{cc}
0 & -\beta_{j+1} \\
1 & a_{j+1}(\zeta)
\end{array}\right] \quad(j \geq 0)
$$


with

$$
\beta_{j+1}:=\frac{\phi_{k_{j}, n_{j}}}{\phi_{k_{j-1}, n_{j-1}}}, \quad a_{j+1}(\zeta):=q_{k_{j}}^{\left(n_{j}\right)}(\zeta)
$$

and initial conditions (4.5).

(4.12) is equivalent to the three-term recurrence of Magnus [Mag62] and Struble [Str63]:

$$
\begin{aligned}
& p_{n_{j}}(\zeta):=a_{j}(\zeta) p_{n_{j-1}}(\zeta)-\beta_{j} p_{n_{j-2}}(\zeta), \quad(j \geq 1) \\
& q_{n_{j}}(\zeta):=a_{j}(\zeta) q_{n_{j-1}}(\zeta)-\beta_{j} q_{n_{j-2}}(\zeta), \quad
\end{aligned}
$$

with the initial conditions (4.5); see also [Gut92] and further references given there.

They correspond to the Magnus $P$-fraction

$$
h_{0}(\zeta)=-\frac{f_{0}(\zeta)}{g_{0}(\zeta)}=\frac{\beta_{1}}{a_{1}(\zeta)}-\frac{\beta_{2}}{a_{2}(\zeta)}-\cdots .
$$

whose partial numerators and denominators are $p_{n_{j}}$ and $q_{n_{j}}$, respectively.

In view of (4.10), the recurrence (3.3) for the residuals becomes

$$
f_{n_{j}}(\zeta)=a_{j}(\zeta) f_{n_{j-1}}(\zeta)-\beta_{j} f_{n_{j-2}}(\zeta) \quad(j \geq 1)
$$

with initial conditions

$$
f_{n_{-1}}(\zeta):=f_{-1}(\zeta):=-g_{0}(\zeta), \quad f_{n_{0}}(\zeta):=f_{0}(\zeta) .
$$

Recall that

$$
\partial f_{n_{j-1}}=-n_{j}>\partial f_{n_{j}}=-n_{j+1}>\partial f_{n_{j+1}}
$$

and

$$
k_{j+1}=\partial a_{j+1}=\partial f_{n_{j-1}}-\partial f_{n_{j}} .
$$

$\beta_{j}$ is just used to make $a_{j+1}$ monic.

This reveals that the P-fraction can be constructed by a simple generalization of the Euclidean algorithm for polynomials. In fact, the Euclidean algorithm for two polynomials $f_{-1}$ and $f_{0}$ with $\partial f_{-1}>\partial f_{0}$, generates a finite sequence of polynomials $f_{j}$ of decreasing degree according to

$$
f_{j}(\zeta):=a_{j}(\zeta) f_{j-1}(\zeta)-\beta_{j} f_{j-2}(\zeta) \quad(j \geq 1),
$$

with $0 \neq \beta_{j} \in \mathbb{C}$ arbitrary and $a_{j} \in \mathcal{P}$. Note that this is the same formula as (4.16) except for a slightly different indexing scheme.

(4.14) and (4.16) define the extended Euclidean algorithm for power series in $\zeta^{-1}$. The word "extended" refers to the fact that not only the residuals $f_{n_{j}}$ are updated, which are the tails of the P-fraction (4.15), but also the corresponding partial numerators and denominators $p_{n_{j}}$ and $q_{n_{j}}$; see Bultheel and Van Barel [BV97] for a detailed discussion of the extended Euclidean algorithm.

In the generic case, where $a_{j}(\zeta)=\zeta-\alpha_{j}$, the Euclidean algorithm for power series is also referred to as Chebyshev algorithm. An alternative generalization of the latter to the case where $k>1$ for some $n$ was proposed by Golub and Gutknecht [GG90].

Dividing (4.16) by $f_{n_{j-1}}(\zeta)$ shows that $a_{j}$ is the polynomial or principal part of the formal Laurent series at $\infty$ of $\beta_{j} f_{n_{j-2}} / f_{n_{j-1}}$. This fact gave rise to the name P-fraction. 


\section{Inner polynomials}

Let us return to the Padé approximation problems for $\left(g_{0}, f_{0}\right)$ or, equivalently, for $h_{0}: \equiv-f_{0} / g_{0}$ that we considered in section 2. If $n$ is not regular, that is, if $\mathbf{M}_{n}$ of (2.7) is singular, there is no or not a unique polynomial $q_{n} \in \mathcal{P}_{n}$ satisfying the corresponding Padé condition (2.4b) with some $p_{n} \in \mathcal{P}_{n-1}$ and some $f_{n} \in \mathcal{L}_{-n-1}^{*}$.

However, there are still solutions to a relaxed Padé condition: if $n$ is regular and $k>0$, we can always find a Padé form

$$
\left(p_{n+k}, q_{n+k}\right) \in \mathcal{P}_{n+k-1} \times \mathcal{P}_{n+k} \text { with } q_{n+k} \text { monic }
$$

such that the corresponding residual $f_{n+k}$ satisfies

$$
f_{n+k}: \equiv g_{0} p_{n+k}+f_{0} q_{n+k} \in \mathcal{L}_{-n-1}^{*} .
$$

The quotient $r_{n+k, n+k}: \equiv p_{n+k} / q_{n+k}$ has been called an underdetermined Padé approximant in [Gut93]. In conjunction with the algorithm of Theorem 3.1, which allows us to compute a sequence of regular indices and pairs, we will refer to those $q_{n+k}$ that are not part of this sequence but still satisfy (5.1)-(5.2) as inner polynomials.

The following theorem, which has been proved in [GG94], establishes not only the well-known existence of such inner polynomials and underdetermined Padé approximants, but also how to compute them from an earlier regular pair.

THEOREM 5.1. Let [ $\left.\grave{q}_{n} q_{n}\right]$ be a normalized nth regular pair for $\left[\begin{array}{ll}g_{0} & f_{0}\end{array}\right]$, and let $k$ be a positive integer. Consider any pair $\left(p_{n+k}, q_{n+k}\right)$ constructed according to

$$
\left[\begin{array}{c}
p_{n+k} \\
q_{n+k}
\end{array}\right]:=\left[\begin{array}{ll}
\grave{p}_{n} & p_{n} \\
\grave{q}_{n} & q_{n}
\end{array}\right]\left[\begin{array}{l}
b \\
a
\end{array}\right]
$$

from a pair

$$
(b, a) \in \mathcal{P}_{k-1} \times \mathcal{P}_{k} \quad \text { with a monic }
$$

that satisfies

$$
f_{n+k}: \equiv g_{n} b+f_{n} a \in \mathcal{L}_{-n-1}^{*} .
$$

Then (5.1) and (5.2) hold.

Condition (5.5) is fulfilled when

$$
b(\zeta):=-\frac{\zeta^{k} f_{n}(\zeta)}{g_{n}(\zeta)}+\mathcal{O}_{-}\left(\zeta^{-1}\right), \quad a(\zeta):=\zeta^{k} ;
$$

that is,

$$
b(\zeta):=\mu_{1}^{(n)} \zeta^{k-1}+\mu_{2}^{(n)} \zeta^{k-2}+\cdots+\mu_{k}^{(n)}
$$

if

$$
h_{n}(\zeta): \equiv-\frac{f_{n}(\zeta)}{g_{n}(\zeta)} \equiv: \mu_{1}^{(n)} \zeta^{-1}+\mu_{2}^{(n)} \zeta^{-2}+\cdots .
$$

The computation of $b(\zeta)$ in (5.6) requires only the solution of a $k \times k$ triangular Toeplitz system, and as $k$ is growing, these systems are nested, so that the solution of the last one contains the solution for all. 


\section{Matrix representations of Padé conditions and orthogonality}

The most relevant aspect of the Padé condition (2.4b) for the Padé form $\left(p_{n}, q_{n}\right)$ is that the terms in $\zeta^{-n}, \ldots, \zeta^{n-1}$ on the left-hand side cancel. If $g_{0}(\zeta) \equiv 1$ as in (2.5), $p_{n} \in \mathcal{P}_{n-1}$ is chosen so that the positive powers cancel, while $q_{n}$ must be chosen so that the $n+1$ terms in $\zeta^{-n}, \ldots, \zeta^{-1}, \zeta^{0}$ cancel. If we choose some fixed $N>n$ and assume that all $n<N$ are regular indices, this condition can be formulated in terms of the $N \times N$ Hankel moment matrix $\mathrm{M}_{N}$ for $h_{0}: \equiv-f_{0} / g_{0}$ as

$$
\mathrm{M}_{N} \mathrm{R}_{N}=\mathrm{F}_{N}
$$

where

$$
\mathrm{R}_{N}: \equiv\left[\begin{array}{ccccc}
1 & \rho_{0,1} & \cdots & \cdots & \rho_{0, N-1} \\
& 1 & \cdots & \cdots & \rho_{1, N-1} \\
& & \ddots & & \vdots \\
& & & \ddots & \rho_{N-2, N-1} \\
& & & & 1
\end{array}\right]
$$

is a unit upper triangular matrix containing in its columns the coefficients of the polynomials $q_{0}, q_{1}, \ldots, q_{N-1}$, while $\mathrm{F}_{N}$ is a lower triangular matrix

$$
\mathrm{F}_{N}: \equiv\left[\begin{array}{cccc}
\phi_{1,0} & & & \\
\phi_{2,0} & \phi_{1,1} & & \\
\vdots & \vdots & \ddots & \\
\phi_{N, 0} & \phi_{N-1,1} & \cdots & \phi_{1, N-1}
\end{array}\right]
$$

whose columns contain the coefficients, down to those of the term in $\zeta^{-N}$, of $f_{0}, f_{1}, \ldots, f_{N-1}$.

If we multiply (6.1) from the left by $\mathrm{R}_{N}^{\top}$, we obtain

$$
\mathrm{R}_{N}^{\top} \mathrm{M}_{N} \mathrm{R}_{N}=\mathrm{R}_{N}^{\top} \mathrm{F}_{N}
$$

where the left-hand side is clearly symmetric and the right-hand side is clearly lower triangular, hence both sides must equal a diagonal matrix $\mathrm{D}_{N}$ : on the left,

$$
\mathrm{R}_{N}^{\top} \mathrm{M}_{N} \mathrm{R}_{N}=\mathrm{D}_{N}: \equiv \operatorname{diag}\left[\delta_{0} \cdots \delta_{N-1}\right] \text {, }
$$

where

$$
\delta_{n}: \equiv \mathbf{r}_{n}^{\top} \mathrm{M}_{n+1} \mathbf{r}_{n}, \quad \mathbf{r}_{n}: \equiv\left[\begin{array}{lll}
\rho_{0, n} & \ldots \rho_{n, n}
\end{array}\right]^{\top} .
$$

Eq. (6.4) is often referred to as an inverse symmetric $L D U$ decomposition of $M_{N}$. On the right, $\mathrm{R}_{N}^{\top} \mathrm{F}_{N}=\mathrm{D}_{N}$, or, $\left(\mathrm{R}_{N}^{\top}\right)^{-1}=\mathrm{F}_{N} \mathrm{D}_{N}^{-1}$, and, therefore, substituting $\mathrm{R}_{N}$ in (6.4) yields a symmetric $L D U$ decomposition of $\mathrm{M}_{N}$ :

$$
\mathrm{M}_{N}=\mathrm{F}_{N} \mathrm{D}_{N}^{-1} \mathrm{~F}_{N}^{\top} \text {. }
$$

The decompositions (6.4) and (6.6) are well known and are fully analogous to decomposition related to the Levinson and Schur algorithms for Toeplitz matrices, except that the latter are not necessarily symmetric; see, for example, [GH95a] for the decompositions of Toeplitz matrices. In particular, (6.4) is just a matrix representation of the mutual formal orthogonality of the FOPs.

If $q_{n}$ is not regular for some $n$, the decompositions (6.4) and (6.6) do not exist in this form. But by applying Theorem 5.1 we can redefine all those $q_{n}$ and $f_{n}$ so that (6.4) and (6.6) hold as a inverse symmetric block LDU decomposition and a 
symmetric block LDU decomposition, respectively. Then, $\mathrm{D}_{N}$ is block diagonal, $\mathrm{R}_{N}$ is still unit upper triangular, and $\mathrm{F}_{n}$ is lower block triangular.

We may make the blocks bigger than theoretically needed and only keep those regular $q_{n}$ that are well-conditioned. Then the columns of $\mathrm{R}_{n}$ and $\mathrm{F}_{n}$ with wellconditioned regular index $n$ still contain the coefficients of the FOPs and the corresponding residuals. They are the first columns of a block, while the other columns contain the coefficients of the inner polynomials and the corresponding residuals, respectively.

If we want to aim at an analogous treatment of the general Padé conditions (2.4a)-(2.4b) for pairs of Padé forms $\left(\grave{p}_{n}, \grave{q}_{n}\right),\left(p_{n}, q_{n}\right)$ that interpolate pairs of power series $\left(g_{0}, f_{0}\right)$, then we need to include the powers $\zeta^{0}, \ldots, \zeta^{n-1}$. Using our notation (3.8) for Sylvester matrices we can write (with $e_{1}$ and $e_{N}$ the first and the last standard unit vector of $\mathbb{C}^{N}$ )

$$
\mathrm{S}_{N}\left(g_{0}, f_{0}\right)\left[\begin{array}{c|c}
\grave{\mathrm{P}}_{N} & \mathrm{P}_{N} \\
\hline \dot{\mathrm{R}}_{N} & \mathrm{R}_{N}
\end{array}\right]=\left[\begin{array}{c|c}
\mathrm{e}_{N}^{\top} \mathrm{e}_{1} & \mathrm{O} \\
\hline \mathrm{G}_{N} & \mathrm{~F}_{N}
\end{array}\right],
$$

where $\mathrm{R}_{N}$ is defined in (6.2) and $\grave{\mathrm{R}}_{N}, \mathrm{P}_{N}$, and $\grave{\mathrm{P}}_{N}$ are analogous upper triangular matrices with the coefficients of the polynomials $\grave{q}_{n}, p_{n}$, and $\grave{p}_{n}(0 \leq n<N)$, respectively, while $\mathrm{G}_{N}$ is a $N \times N$ lower Hessenberg matrix that contains in its columns the coefficients (down to those of $\zeta_{-N}$ ) of the residuals $g_{n}$ (except that $\gamma_{0,0}=1$ is missing at the top of the first column), and $\mathrm{F}_{N}$ is a $N \times N$ lower triangular matrix with the coefficients of the residuals $f_{n}$ as before.

However, compared to these residuals, those whose coefficients are in $\mathrm{F}_{N}$ of (6.3) are devided by $-g_{0}$, because this is how the general case of $(2.4 \mathrm{a})-(2.4 \mathrm{~b})$ is related to the special case of (2.5). Therefore, to return from (6.7) to the special case (6.1), we multiply (6.7) from the left by a $2 N \times 2 N$ lower triangular Toeplitz matrix that contains the first $2 N$ coefficients of the formal power series of $-1 / g_{0}$. Then, after redefining $F_{N}$, the $(2,2)$ block of the resulting block matrix identity yields exactly (6.1).

\section{Matrix form of the extended Euclidean algorithm}

Now, we can finally aim at the intended reformulation of the extended Euclidean algorithm as a matrix product applied to the Sylvester matrix with the given data. First, we discuss a single step and return to (3.3),

$$
\left[\begin{array}{ll}
g_{n} & f_{n}
\end{array}\right]\left[\begin{array}{cc}
\grave{b} & b \\
\grave{a} & a
\end{array}\right]=\left[\begin{array}{ll}
g_{n+k} & f_{n+k}
\end{array}\right],
$$

and its matrix form (3.11), rewritten with two additional columns and two additional rows as

$$
\mathrm{S}_{k+1}\left(g_{n}, f_{n}\right)\left[\begin{array}{c|c}
\grave{\mathrm{b}} & \mathrm{b} \\
0 & 0 \\
\hline \grave{\mathrm{a}} & \dot{\mathrm{a}} \\
0 & 1
\end{array}\right]=\left[\begin{array}{cc}
\mathrm{e}_{2 k+1,2 k+1} & \mathrm{o}_{2 k+1} \\
\gamma_{1, n+k} & \phi_{1, n+k}
\end{array}\right]
$$

(where $\mathrm{o}_{2 k+1}$ is the zero vector in $\mathbb{C}^{2 k+1}$ ). This corresponds to the terms from $\zeta^{-n+k}$ downto $\zeta^{-n-k-1}$ of (3.3). We can take into account as many leading terms as we want by adding additional rows to the Sylvester matrix and to the right-hand side. In particular, if $g_{n} \in \mathcal{L}_{-n}^{*}$ and $f_{n} \in \mathcal{L}_{-n-1}^{*}$ are polynomials in $\zeta^{-1}$ of degree 
at most $2 N$, we can process all their coefficients if the Sylvester matrix is of size $(2 N-n+2) \times(2 k+2)$.

Starting from a pair

$$
f_{0} \in \mathcal{L}_{-2 N:-1}, \quad g_{0} \in \mathcal{L}_{-2 N: 0}
$$

with $f_{0} \neq 0$ and $g_{0}(\infty): \equiv \gamma_{0,0}=1$, we will apply (3.3) recursively until we reach some $f_{n} \in \mathcal{L}_{-N-1}^{*}$. This will happen for some regular $n \equiv: n_{J} \leq N$. We let $\left\{n_{j}\right\}_{j=0}^{J}$ be the corresponding sequence of regular indices, starting with $n_{0}=0$, and set

$$
k_{j}:=n_{j+1}-n_{j} \quad(j \geq 1) .
$$

Then, $\phi_{k_{j}, n_{j}} \neq 0(\forall j)$. Additionally, we set $n_{-1}:=-1, k_{0}:=1$. The sequence $\left\{n_{j}\right\}_{j=0}^{J}$ need not contain all regular indices, but only the chosen well-conditioned ones.

Adding further terms of order $\mathcal{O}_{-}(-2 N-1)$ to $f_{0}$ and $g_{0}$ will not change the resulting pair $\left(p_{n_{J}}, q_{n_{J}}\right)$.

If, in the step starting at $n=n_{j}$, we write $\grave{a} \equiv: \grave{a}_{j}, \grave{b} \equiv: \grave{b}_{j}$, à $\equiv: \grave{a}_{j}, \grave{\mathrm{b}} \equiv: \grave{\mathrm{b}}_{j}$, etc., then (3.3) and (3.6) yield

$$
\left[\begin{array}{ll}
g_{n_{j+1}} & f_{n_{j+1}}
\end{array}\right]:=\left[\begin{array}{ll}
g_{n_{j}} & f_{n_{j}}
\end{array}\right]\left[\begin{array}{ll}
\grave{b}_{j} & b_{j} \\
\grave{a}_{j} & a_{j}
\end{array}\right] \quad(j \geq 0),
$$

and

$$
\left[\begin{array}{ll}
\grave{p}_{n_{j+1}} & p_{n_{j+1}} \\
\grave{q}_{n_{j+1}} & q_{n_{j+1}}
\end{array}\right]:=\left[\begin{array}{cc}
\grave{p}_{n_{j}} & p_{n_{j}} \\
\grave{q}_{n_{j}} & q_{n_{j}}
\end{array}\right]\left[\begin{array}{cc}
\grave{b}_{j} & b_{j} \\
\grave{a}_{j} & a_{j}
\end{array}\right] \quad(j \geq 0) .
$$

Consequently,

$$
\left[\begin{array}{ll}
g_{n_{J}} & f_{n_{J}}
\end{array}\right]:=\left[\begin{array}{ll}
g_{n_{0}} & f_{n_{0}}
\end{array}\right] \prod_{j=0}^{J-1}\left[\begin{array}{cc}
\grave{b}_{j} & b_{j} \\
\grave{a}_{j} & a_{j}
\end{array}\right]
$$

and

$$
\left[\begin{array}{ll}
\grave{p}_{n_{J}} & p_{n_{J}} \\
\grave{q}_{n_{J}} & q_{n_{J}}
\end{array}\right]:=\left[\begin{array}{ll}
\grave{p}_{n_{0}} & p_{n_{0}} \\
\grave{q}_{n_{0}} & q_{n_{0}}
\end{array}\right] \prod_{j=1}^{J-1}\left[\begin{array}{ll}
\grave{b}_{j} & b_{j} \\
\grave{a}_{j} & a_{j}
\end{array}\right]
$$

with $f_{n_{J}} \in \mathcal{L}_{-N-1}^{*}$. Obviously, we could combine the two equations (7.4) and (7.5) into one.

Let us again look first at the generic case, where $k=1$ for all $n$, and thus $n_{j}=n$. Here, (7.1) reduces to

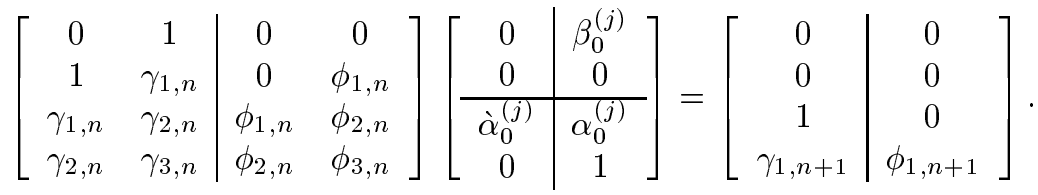

This implies trivially that

$$
\left[\begin{array}{cc|cc}
0 & 1 & 0 & 0 \\
1 & \gamma_{1, n} & 0 & \phi_{1, n} \\
\gamma_{1, n} & \gamma_{2, n} & \phi_{1, n} & \phi_{2, n} \\
\gamma_{2, n} & \gamma_{3, n} & \phi_{2, n} & \phi_{3, n}
\end{array}\right]\left[\begin{array}{cc|cc}
1 & 0 & 0 & \beta_{0}^{(j)} \\
0 & 0 & 0 & 0 \\
\hline 0 & \grave{\alpha}_{0}^{(j)} & 1 & \alpha_{0}^{(j)} \\
0 & 0 & 0 & 1
\end{array}\right]=\left[\begin{array}{cc|cc}
0 & 0 & 0 & 0 \\
1 & 0 & 0 & 0 \\
\gamma_{1, n} & 1 & \phi_{1, n} & 0 \\
\gamma_{2, n} & \gamma_{1, n+1} & \phi_{2, n} & \phi_{1, n+1}
\end{array}\right]
$$

Note that the two additional columns in the second matrix allow us to "store" the coefficients of the residuals in the resulting matrix on the right-hand side. 
To obtain a representation that can accommodate all $N$ steps we need to "imbed" this relationship into $2 N \times 2 N$ matrices. For example, in the first step, where $n=0$, we get

$$
\mathrm{S}_{N}\left(g_{0}, f_{0}\right) \mathrm{C}_{1}=\mathrm{V}_{1}
$$

with

$$
\begin{aligned}
& \mathrm{S}_{N}\left(g_{0}, f_{0}\right): \equiv\left[\begin{array}{cccc|cccc}
0 & \cdots & 0 & 1 & 0 & \cdots & \cdots & 0 \\
\vdots & . \cdot & 1 & \gamma_{1,0} & \vdots & & 0 & \phi_{1,0} \\
0 & . \cdot & . \cdot & \vdots & \vdots & . \cdot & . \cdot & \vdots \\
1 & . & & \vdots & 0 & . \cdot & & \vdots \\
\gamma_{1,0} & & & \gamma_{N, 0} & \phi_{1,0} & & & \phi_{N, 0} \\
\vdots & & . & \vdots & \vdots & & . \cdot & \vdots \\
\vdots & . & & \vdots & \vdots & . \cdot & & \vdots \\
\gamma_{N, 0} & \ldots & \ldots & \gamma_{2 N-1,0} & \phi_{N, 0} & \ldots & \ldots & \phi_{2 N-1,0}
\end{array}\right], \\
& C_{1}: \equiv\left[\begin{array}{cccc|cccc}
1 & 0 & & & 0 & \beta_{0}^{(0)} & & \\
& 0 & \ddots & & & 0 & \ddots & \\
& & \ddots & 0 & & & \ddots & \beta_{0}^{(0)} \\
& & & 0 & & & & 0 \\
\hline 0 & \dot{\alpha}_{0}^{(0)} & & & 1 & \alpha_{0}^{(0)} & & \\
& 0 & \ddots & & & 1 & \ddots & \\
& & \ddots & \grave{\alpha}_{0}^{(0)} & & & \ddots & \alpha_{0}^{(0)} \\
& & & 0 & & & & 1
\end{array}\right]
\end{aligned}
$$

$$
\mathrm{V}_{1}: \equiv\left[\begin{array}{c|ccc|c|ccc}
0 & 0 & \ldots & 0 & 0 & 0 & \ldots & 0 \\
\vdots & \vdots & . \cdot & 0 & \vdots & \vdots & . \cdot & 0 \\
0 & 0 & . \cdot & 1 & \vdots & 0 & . \cdot & 0 \\
1 & 0 & . \cdot & \gamma_{1,1} & 0 & 0 & . \cdot & \phi_{1,1} \\
\gamma_{1,0} & 1 & . & \vdots & \phi_{1,0} & 0 & . \cdot & \vdots \\
\vdots & \gamma_{1,1} & & \vdots & \vdots & \phi_{1,1} & & \vdots \\
\vdots & \vdots & & \vdots & \vdots & \vdots & & \vdots \\
\gamma_{N, 0} & \gamma_{N-1,1} & \ldots & \gamma_{2 N-3,1} & \phi_{N, 0} & \phi_{N-1,1} & \ldots & \phi_{2 N-3,1}
\end{array}\right]
$$

In the second step,

$$
\mathrm{V}_{1} \mathrm{C}_{2}=\mathrm{V}_{2} \text {, }
$$


$\mathrm{C}_{2}$ will have two $2 \times 2$ unit matrices where $\mathrm{C}_{1}$ had two 1 's, and therefore the columns with indices $1,2, N+1, N+2$ of $\mathrm{V}_{1}$ will remain untouched in the transition to $\mathrm{V}_{2}$ :

$$
\mathrm{V}_{2}=\left[\begin{array}{cc|ccc|cc|ccc}
0 & 0 & 0 & \ldots & 0 & 0 & 0 & 0 & \ldots & 0 \\
\vdots & \vdots & \vdots & . \cdot & \vdots & \vdots & \vdots & \vdots & . & \vdots \\
0 & 0 & 0 & & 0 & \vdots & 0 & 0 & & \vdots \\
1 & 0 & \vdots & . \cdot & 1 & 0 & \vdots & \vdots & & 0 \\
\gamma_{1,0} & 1 & 0 & . \cdot & \gamma_{1,2} & \phi_{1,0} & 0 & \vdots & . \cdot & \phi_{1,2} \\
\vdots & \gamma_{1,1} & 1 & . \cdot & \vdots & \vdots & \phi_{1,1} & 0 & . \cdot & \vdots \\
\vdots & \vdots & \gamma_{1,2} & & \vdots & \vdots & \vdots & \phi_{1,2} & & \vdots \\
\vdots & \vdots & \vdots & & \vdots & \vdots & \vdots & \vdots & & \vdots \\
\gamma_{N, 0} & \gamma_{N-1,1} & \gamma_{N-2,2} & \ldots & \gamma_{2 N-5,2} & \phi_{N, 0} & \phi_{N-1,1} & \phi_{N-2,2} & \ldots & \phi_{2 N-5,2}
\end{array}\right],
$$

while all the Toeplitz blocks in $C_{2}$ and $V_{2}$ are by one column and one or two rows smaller than the corresponding ones in $\mathrm{C}_{1}$ and $\mathrm{V}_{1}$.

By now it should be clear, how, in the generic case, the matrices $\mathrm{V}_{n}$ change from step to step until at the end,

$$
\mathrm{V}_{N}=\left[\begin{array}{c|c}
\mathrm{e}_{N}^{\top} \mathrm{e}_{1} & \mathrm{O} \\
\hline \mathrm{G}_{N} & \mathrm{~F}_{N}
\end{array}\right]
$$

is the matrix on the right-hand side of (6.7).

Now, we turn to the look-ahead case where $n=n_{j}$ and $k=k_{j}>1$, either due to the situation (4.8) or because of a general look-ahead step that also leaves out ill-conditioned regular pairs. We append $N-n_{j}-k_{j}-1=N-n_{j+1}-1$ rows at the bottom of the $\left(2 k_{j}+2\right) \times\left(2 k_{j}+2\right)$ matrix $S_{k_{j}+1}\left(g_{n_{j}}, f_{n_{j}}\right)$ to get the $\left(N-n_{j}+k_{j}+1\right) \times\left(2 k_{j}+2\right)$ matrix

$$
\underline{\mathrm{S}}_{k_{j}+1}\left(g_{n_{j}}, f_{n_{j}}\right): \equiv
$$

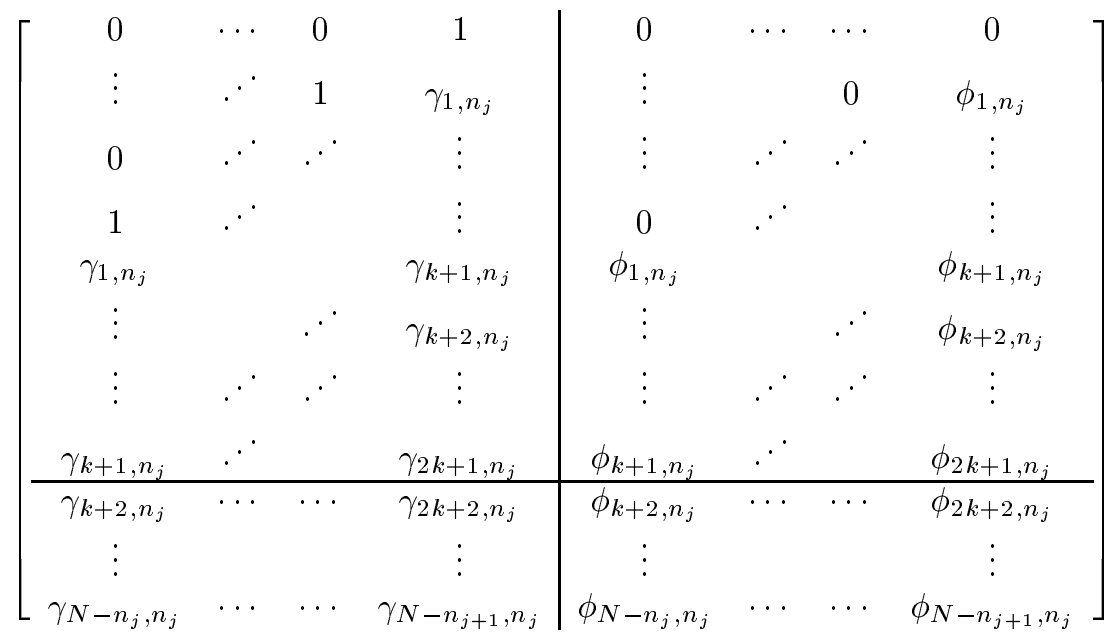


(for simplicity, we write $k$ instead of $k_{j}$ in the indices). Now, (7.1) reads

$$
\underline{S}_{k_{j}+1}\left(g_{n_{j}}, f_{n_{j}}\right)\left[\begin{array}{c|c}
0 & \beta_{0}^{(j)} \\
\grave{\beta}_{1}^{(j)} & \beta_{1}^{(j)} \\
\vdots & \vdots \\
\grave{\beta}_{k-1}^{(j)} & \beta_{k-1}^{(j)} \\
0 & 0 \\
\hline \grave{\alpha}_{0}^{(j)} & \alpha_{0}^{(j)} \\
\grave{\alpha}_{1}^{(j)} & \alpha_{1}^{(j)} \\
\vdots & \vdots \\
\grave{\alpha}_{k-1}^{(j)} & \alpha_{k-1}^{(j)} \\
0 & 1
\end{array}\right]=\left[\begin{array}{c|c}
0 & 0 \\
0 & 0 \\
\vdots & \vdots \\
\vdots & \vdots \\
0 & 0 \\
1 & 0 \\
\gamma_{1, n_{j+1}} & \phi_{1, n_{j+1}} \\
\hline \gamma_{2, n_{j+1}} & \phi_{2, n_{j+1}} \\
\vdots & \vdots \\
\gamma_{N-n_{j}-k, n_{j+1}} & \phi_{N-n_{j}-k, n_{j+1}}
\end{array}\right] .
$$

This system contains $N-n_{j+1}+1$ equations and reflects the terms from $\zeta^{-n_{j+1}-1}$ downto $\zeta^{-N+1}$ of (3.3). We could add $N+n_{j}-k_{j}-1$ zero rows at the top to get a total of $2 N$ rows and to cover the terms upto $\zeta^{N}$.

Next we want to include the inner polynomials. To this end, we multiply the $\left(N-n_{j}+k_{j}+1\right) \times\left(2 k_{j}+2\right)$ matrix $\underline{\mathrm{S}}_{k_{j}+1}\left(g_{n_{j}}, f_{n_{j}}\right)$ by the $\left(2 k_{j}+2\right) \times\left(2 k_{j}+2\right)$ matrix

$$
\mathrm{C}_{j}^{\circ}: \equiv\left[\begin{array}{cc}
\grave{\mathrm{B}_{j}^{\circ}} & \mathrm{B}_{j}^{\circ} \\
\hline \grave{\mathrm{A}}_{j}^{\circ} & \mathrm{A}_{j}^{\circ}
\end{array}\right]: \equiv\left[\begin{array}{ccccc|ccccc}
1 & 1 & \cdots & 1 & 0 & 0 & \mu_{1}^{(j)} & \cdots & \mu_{k}^{(j)} & \beta_{0}^{(j)} \\
0 & 0 & \cdots & 0 & \grave{\beta}_{1}^{(j)} & & 0 & \ddots & \vdots & \beta_{1}^{(j)} \\
\vdots & & & \vdots & \vdots & & & \ddots & \mu_{1}^{(j)} & \vdots \\
\vdots & & & \vdots & \grave{\beta}_{k-1}^{(j)} & & & & 0 & \beta_{k-1}^{(j)} \\
0 & 0 & \cdots & 0 & 0 & & & & & 0 \\
\hline 0 & 0 & \cdots & 0 & \grave{\alpha}_{0}^{(j)} & 1 & 0 & \cdots & 0 & \alpha_{0}^{(j)} \\
\vdots & & & \vdots & \grave{\alpha}_{1}^{(j)} & & 1 & \ddots & \vdots & \alpha_{1}^{(j)} \\
\vdots & & & \vdots & \vdots & & & \ddots & 0 & \vdots \\
\vdots & & & \vdots & \grave{\alpha}_{k-1}^{(j)} & & & & 1 & \alpha_{k-1}^{(j)} \\
0 & 0 & \cdots & 0 & 0 & & & & 1
\end{array}\right] .
$$

This yields 


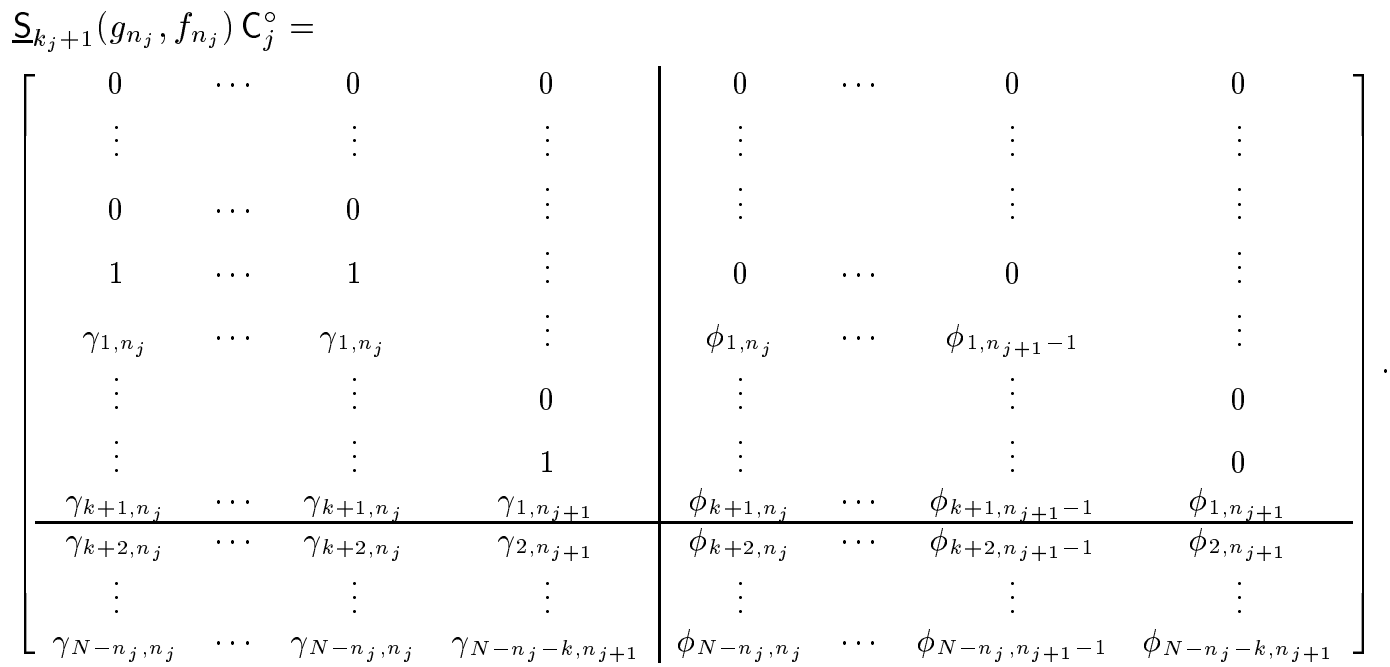

We still need to imbed these matrices into even bigger ones of size $2 N \times 2 N$. First, as mentioned, $\underline{\mathrm{S}}_{k_{j}+1}\left(g_{n_{j}}, f_{n_{j}}\right)$ is extended on top by $N+n_{j}-k_{j}-1$ zero rows to make it a $2 N \times\left(2 k_{j}+2\right)$ matrix. Then each half of it is extended on the right by $N-n_{j}-k_{j}-1$ columns, so that each half has size $2 N \times\left(N-n_{j}\right)$. Finally, at the left of each half, $n_{j}$ columns with the previously computed coefficients of the residuals $g_{n}, n=0, \ldots n_{j}-1$, and $f_{n}, n=0, \ldots n_{j}-1$, are appended. This yields a $2 N \times 2 N$ matrix that we call again $\mathrm{V}_{j-1}$. At the beginning and at the end, we have, respectively,

$$
\mathrm{V}_{0}=\mathrm{S}_{N}\left(g_{0}, f_{0}\right), \quad \mathrm{V}_{J}=\left[\begin{array}{c|c}
\mathrm{e}_{N}^{\top} \mathrm{e}_{1} & \mathrm{O} \\
\hline \mathrm{G}_{N} & \mathrm{~F}_{N}
\end{array}\right],
$$

where the last matrix is the same one that appears in (6.7).

To imbed $C_{j}^{\circ}$, each of the four square blocks of size $k_{j}+1$ of $\mathrm{C}_{j}^{\circ}$ is first extended to a square block of size $N-n_{j}+1$ by appending $N-n_{j}-k_{j}$ rows and columns, so that the last $N-n_{j}-k_{j}+1$ columns have Toeplitz structure. This yields the blocks $\grave{B}_{j}, \mathrm{~B}_{j}, \grave{\mathrm{A}}_{j}$, and $\mathrm{A}_{j}$, which are imbedded into a $2 N \times 2 N$ matrix $\mathrm{C}_{j}$ as follows:

$$
\mathrm{C}_{j}: \equiv\left[\begin{array}{cc|cc}
\mathrm{I}_{n_{j}-1} & \mathrm{O} & \mathrm{O}_{n_{j}-1} & \mathrm{O} \\
\mathrm{O} & \grave{\mathrm{B}}_{j} & \mathrm{O} & \mathrm{B}_{j} \\
\hline \mathrm{O}_{n_{j}-1} & \mathrm{O} & \mathrm{I}_{n_{j}-1} & \mathrm{O} \\
\mathrm{O} & \grave{\mathrm{A}}_{j} & \mathrm{O} & \mathrm{A}_{j}
\end{array}\right] .
$$

Note that in view of the 1's in the upper left corners of $\dot{B}_{j}$ and $\mathrm{A}_{j}$ the blocks $\mathrm{I}_{n_{j}-1}$ are part of unit matrices of size $n_{j}$, whose boundaries do not match those of the blocks $\grave{\mathrm{B}}_{j}, \grave{\mathrm{A}}_{j}, \mathrm{~B}_{j}$, and $\mathrm{A}_{j}$, however. Now we have

$$
\mathrm{V}_{j-1} \mathrm{C}_{j}=\mathrm{V}_{j}
$$

and, consequently,

$$
\mathrm{S}_{N}\left(g_{0}, f_{0}\right) \prod_{j=1}^{J} \mathrm{C}_{j}=\left[\begin{array}{c|c}
\mathrm{e}_{N}^{\top} \mathrm{e}_{1} & \mathrm{O} \\
\hline \mathrm{G}_{N} & \mathrm{~F}_{N}
\end{array}\right]
$$


where $G_{N}$ is now lower block Hessenberg, and $F_{N}$ is lower block triangular. So, finally, the comparison with (6.7) reveals that

$$
\prod_{j=1}^{J} \mathrm{C}_{j}=\left[\begin{array}{l|l}
\grave{\mathrm{P}}_{N} & \mathrm{P}_{N} \\
\hline \grave{\mathrm{R}}_{N} & \mathrm{R}_{N}
\end{array}\right]
$$

Eqs. (7.8)-(7.12) are the matrix relations we have been looking for. Eq. (7.10) describes a single step of the Euclidean algorithm as a matrix multiplication, and (7.11) summarizes the whole process as a matrix product with the factors $C_{j}$. Moreover, (7.11) shows how the coefficients of the Padé forms constructed are also obtained by multiplying all these matrices $C_{j}$. Finally, as we have seen at the end of section 6 , it is an easy matter to link (7.8) to the block LDU decomposition and to the inverse block LDU decomposition of the Hankel matrix $\mathrm{M}_{n}$.

Acknowledgement. The author would like to thank Paul van Dooren for pointing out some references to earlier related work such as [HS69]. He also mentioned that his diploma student Paul Bailey wrote a related thesis, which, however, was not available to the author.

\section{References}

[Bec96] B. Beckermann, The stable computation of formal orthogonal polynomials, Numerical Algorithms 11 (1996), 1-23.

[BV97] A. Bultheel and M. Van Barel, Linear algebra, rational approximation and orthogonal polynomials, Elsevier, Amsterdam, 1997.

[CM93] S. Cabay and R. Meleshko, A weakly stable algorithm for Padé approximants and the inversion of Hankel matrices, SIAM J. Matrix Anal. Appl. 14 (1993), 735-765.

[DI89] J.-M. Delosme and I. C. F. Ipsen, From Bareiss' algorithm to the stable computation of partial correlations, J. Comput. Appl. Math. 27 (1989), 53-91.

[GG90] G. H. Golub and M. H. Gutknecht, Modified moments for indefinite weight functions, Numer. Math. 57 (1990), 607-624.

[GG94] W. B. Gragg and M. H. Gutknecht, Stable look-ahead versions of the Euclidean and Chebyshev algorithms, Approximation and Computation (R. V. M. Zahar, ed.), ISNM, vol. 119, Birkhäuser Verlag, Basel-Boston-Berlin, 1994, pp. 231-260.

[GGWY82] W. B. Gragg, F. G. Gustavson, D. D. Warner, and D. Y. Y. Yun, On fast computation of superdiagonal Padé fractions, Math. Programming Stud. 18 (1982), 39-42.

[GH95a] M. H. Gutknecht and M. Hochbruck, Look-ahead Levinson and Schur algorithms for non-Hermitian Toeplitz systems, Numer. Math. 70 (1995), 181-227.

[GH95b] M. H. Gutknecht and M. Hochbruck, The stability of inversion formulas for Toeplitz matrices, Linear Algebra Appl. 223/224 (1995), 307-324.

[Gut92] M. H. Gutknecht, A completed theory of the unsymmetric Lanczos process and related algorithms, Part I, SIAM J. Matrix Anal. Appl. 13 (1992), 594-639.

[Gut93] M. H. Gutknecht, Stable row recurrences in the Padé table and generically superfast lookahead solvers for non-Hermitian Toeplitz systems, Linear Algebra Appl. 188/189 (1993), 351-421.

[HR84] G. Heinig and K. Rost, Algebraic methods for Toeplitz-like matrices and operators, Akademie-Verlag, Berlin, and Birkhäuser, Basel/Stuttgart, 1984.

[HS69] A. S. Householder and G. W. Stewart, Bigradients, Hankel determinants, and the Padé table, Constructive aspects of the fundamental theorem of algebra (B. Dejon and P. Henrici, eds.), Wiley-Interscience, 1969, pp. 131-150.

[Mag62] A. Magnus, Certain continued fractions associated with the Padé table, Math. Z. 78 (1962), 361-374

[Str63] G. W. Struble, Orthogonal polynomials: variable-signed weight functions, Numer. Math 5 (1963), 88-94. 